\title{
A||| publications
}

an Open Access Journal by MDPI

\begin{tabular}{c|c} 
Tracked for & CITESCORE \\
Impact & $\mathbf{3 . 5}$ \\
Factor &
\end{tabular}

D Academic Open Access Publishing

since 1996 


\section{publications}

an Open Access Journal by MDPI

Editor-in-Chief

Dr. Gemma. E. Derrick

\section{Message from the Editor-in-Chief}

As the Editor-in-Chief of Publications (ISSN 23046775), I warmly invite you to submit your research for consideration for publication in our journal. Publications is an international, peer reviewed, fully Open Access journal that provides an advanced forum for research studies related to all aspects of scholarly publishing. While primarily a vehicle for research and review articles, we will also publish, from time to time, case studies and opinion pieces. We aim to offer high-quality, responsible services and rapid decision-making so that submitting and publishing with us should be a rewarding experience. If you wish to discuss your ideas for submission in advance, or if you have topics for potential Special Issues you would like us to consider, or indeed have any queries about any other aspect of Publications, then please do not hesitate to contact me personally at: g.derrick@lancaster.ac.uk

\section{Author Benefits}

Open Access Unlimited and free access for readers

C No Copyright Constraints Retain copyright of your work and free use of your article

\section{\&. Thorough Peer-Review}

Coverage by Leading Indexing Services Scopus, ESCl (Web of Science), RePEc, dblp and other databases

I] No Space Constraints, No Extra Space or Color Charges No restriction on the length of the papers, number of figures or colors 


\section{Aims and Scope}

Publications (ISSN 2304-6775) is an international, peerreviewed, fully open access journal that provides a home for research related to all aspects of scholarly publication and culture. Scholarly publishing is the gateway to the academic record, and it should be studied and critically analysed in all its dimensions with the same rigour expected of other research. Publications aims to provide an interdisciplinary forum for scholars, publishing professionals, librarians, funders, innovative service providers and others to communicate original research on a range of topics that address current issues and inform best practice.

The scope of Publications includes:

Theory and practices of scholarly communication

Public access to and engagement with scientific research Globalisation of research and the advent of open science The social, legal and ethical dimensions of publication Digitisation and other innovations in scholarly publishing technologies

Metadata, standards, infrastructures and linking in the scholarly record

Financial models and sustainability issues for scholarly publishing

Copyright, licensing and other legal issues in publishing Issues related to peer review and academic governance practices

Critical bibliometric and scientometric studies, including socio-technical perspectives

etc

\section{Editorial Office}

Publications Editorial Office publications@mdpi.com MDPI, St. Alban-Anlage 66 4052 Basel, Switzerland Tel: +41616837734 www.mdpi.com mdpi.com/journal/publications 


\section{MDPI is a member of}

\section{CASPA}

The Association of Learned \& Profession Society Publishers

\section{Crossref \\ STM'}

Society

for Scholarly

Publishing
$|\mathbf{C}| \mathbf{O}|\mathbf{P}| \mathbf{E} \mid$

SPARC*

Europe

\section{DOAJ}

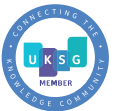

ORCID

\section{Follow}

f facebook.com/MDPIOpenAccessPublishing

twitter.com/MDPIOpenAccess

in linkedin.com/company/mdpi

(O) instagram.com/mdpiopenaccess

6) weibo.com/mdpicn

(2) Wechat: MDPI-China

\section{Subscribe}

blog.mdpi.com 